\title{
Idiopathic Orbital Myositis Revisited
}

\author{
Ilse Mombaerts ${ }^{1}$ - Alan A. McNab ${ }^{2}$
}

Accepted: 18 November 2021 / Published online: 9 February 2022

(c) The Author(s), under exclusive licence to Springer Science+Business Media, LLC, part of Springer Nature 2022

\begin{abstract}
Purpose of Review To review current knowledge regarding idiopathic orbital myositis.

Recent Findings Recent publications have focused on possible causes of orbital myositis and the process to reach a diagnosis of idiopathic orbital myositis. With inflamed and enlarged extraocular muscles, features to distinguish between competing diagnostic possibilities are based on imaging in the context of history and clinical signs. Idiopathic orbital myositis is characterized by the clinical triad of acute onset of orbital pain exacerbated on eye movement, double vision, and redness or swelling of the eyelids or conjunctiva, along with the radiological finding of homogeneous, fusiform enlargement of one or more extraocular muscles. In atypical or inconclusive clinico-radiological findings for a diagnosis of idiopathic orbital myositis, or where the clinical behavior changes or fails to respond to corticosteroid treatment, a systemic and oncologic work-up and muscle biopsy are warranted to exclude specific local or systemic disease as cause of the inflamed and enlarged muscle. Summary As our understanding of idiopathic orbital myositis evolves, the diagnostic focus is shifting toward earlier identification of underlying local or systemic disease through systemic work-up and muscle biopsy.
\end{abstract}

Keywords Orbital myositis $\cdot$ Idiopathic orbital inflammation $\cdot$ Extraocular muscle $\cdot$ Muscle biopsy $\cdot$ Corticosteroids

\section{Introduction}

The eye is surrounded by seven extraocular muscles (EOMs). Four rectus (two horizontal and two vertical) and two oblique muscles control the movement of the eye, and one, the superior palpebral levator muscle, controls the elevation of the upper eyelid. Compared to non-skeletal muscles, the EOMs have different structural, functional, biochemical, and immunological properties, rendering them distinct diseasepropensity characteristics $[1,2]$.

Orbital myositis is a general term for inflamed EOMs and includes systemic inflammatory, auto-immune and infective disease, drug reactions, and post-streptococcal pharyngeal infection and paraneoplastic immune phenomena as possible causes $[3 \bullet \bullet]$. Within the concept of orbital myositis,

This article is part of the Topical Collection on Vasculitis

Ilse Mombaerts

ilse.mombaerts@uzleuven.be

1 Orbital Unit, Department of Ophthalmology, University Hospitals Leuven, Herestraat 49, 3000 Leuven, Belgium

2 Orbital, Plastic and Lacrimal Clinic, Royal Victorian Eye and Ear Hospital, Melbourne, Australia idiopathic orbital myositis (IOM) constitutes a separate entity and is the commonest form. Skeletal polymyositis and dermatomyositis are usually not associated with orbital myositis. Ocular myositis has become an obsolete term for orbital myositis.

The earliest description of IOM dates back to 1903 when Gleason reported a patient with clinical symptoms of a rapidly progressing bilateral orbital tumor with histopathological findings of inflamed EOMs [4]. In 1905, Birch-Hirschfeld described a series of patients presenting with clinical signs of an orbital mass lesion consisting of fibro-inflammatory tissue, and coined the term orbital pseudotumor, nowadays referred to as idiopathic or non-specific orbital inflammation [5]. IOM belongs to the spectrum of idiopathic orbital inflammation [6].

\section{Clinical Features}

Females are more often affected by IOM, by a ratio of 1.4:1 $[7,8]$. IOM can occur at any age (range 12 to 78 years) but the majority are young adults (mean; 33, 41, and 43 years, from separate studies) [7-9]. IOM may arise during or after pregnancy, although a causal relationship is not established 
[10]. The disease is associated with a symptom triad of acute onset: (i) orbital pain, which is exacerbated by eye movement and particularly worse in the direction of action and stretching of the muscle; (ii) double vision due to limited motility of the eye; and (iii) eyelid or conjunctival swelling or redness, in particular conjunctival injection around the insertion of the affected muscle [11] (Fig. 1).

Most frequently IOM affects a single muscle $(88 \%$ in one series) [8]. Other patterns include two affected muscles, or three or more muscles. Bilateral involvement occurs in $8-20 \%$ of cases, but becomes more common (50\%) synchronously or metachronously in recurrent disease $[7,8$, 12]. The relative frequency of involvement of each EOM is medial rectus $>$ lateral rectus $>$ superior rectus $>$ inferior rectus $>$ obliques $>$ levator. Patients may suffer from acute or subacute, single, or multiple flares in which case the same or contralateral muscle is often affected [8]. When different muscles become involved in recurrent disease, it is referred to as migratory IOM [13]. Rarely, optic nerve dysfunction develops from compression of the enlarged muscle in the orbital apex [12].

Ocular motility varies over the course of the disease and between patients. A minority may have normal motility but with pain in the direction of action of the muscle. Most patients experience double vision from a paretic motility disturbance, implying limitation in the direction of action of the affected muscle [9, 12] (Fig. 1). In levator myositis, paresis manifests as upper eyelid ptosis. However, ptosis may also result from eyelid edema in IOM without levator muscle affection.

A mixed paretic and restrictive pattern, or a pure restrictive pattern (implying limitation in the opposite direction of action of the affected muscle) is also observed. Where both horizontal or vertical muscles, or all muscles are affected, paresis and restriction are difficult to determine based on clinical motility alone, in which case forced duction testing can be conducted. In 1994, Siatkowski attributed the different strabismus patterns of the affected horizontal muscles to duration of the inflammatory process: entering the paretic phase from 7 days of onset of disease, acquiring an additional restrictive component in the following weeks, and finally resulting in pure restriction [14]. Such a change of motility disturbance over time, however, is not always seen in IOM. This inconsistency may be explained by the fact that Siatkowski's series of 75 patients with IOM were diagnosed with ultrasound imaging only, and included 13 patients (17\%) with different diagnosis than IOM (7 cases had other orbital structures affected and 6 had Graves' orbitopathy) [14].

An extended period of restrictive movement would suggest fibrosis in the muscle, in which case recovery may be incomplete. On the other hand, permanent paresis often develops and presumably results from muscle fiber destruction caused by chronic inflammation [12, 15•]. Importantly, a paretic pattern of an enlarged muscle is not pathognomonic for IOM and can also be encountered in other diseases affecting the EOM such as lymphoproliferative disease, giant cell orbital myositis, and systemic lupus erythematosus [16]. Likewise, restrictive strabismus is not typical of IOM and occurs more often in Graves' orbitopathy (GO), but may also occur in metastasis to the EOM [16]. Of interest, a mixed paretic and restrictive pattern may also arise in a massively swollen EOM from metastatic origin, with the weakened muscle function resulting from the mass effect [16].

Another useful but rarely reported clinical sign is the presence of globe retraction with eye movement [17]. The sign, originally described by Osher, may be subtle and needs to be specifically looked for where the horizontal muscles are affected [18]. It is best elicited by first examining horizontal eye movements with the patient facing the examiner and checking for narrowing of the vertical palpebral fissure, and then repeating eye movements with the patient in profile and looking for retraction of the globe, which usually occurs when the eye is looking in a direction opposite to the direction of action of the involved muscle (Fig. 1). However, acquired orbital retraction syndrome is not a pathognomonic
Fig. 1 Patient with acute idiopathic orbital myositis. (Top left) Redness at the lateral sector of the left eye. (Top right) Limitation of the left eye motility in abduction. (Bottom left) Globe retraction with narrowing of the vertical palpebral fissure in adduction. (Bottom right) Magnetic resonance imaging of the orbit, axial view, showing homogeneous and fusiform enlargement of the belly of the left lateral rectus muscle
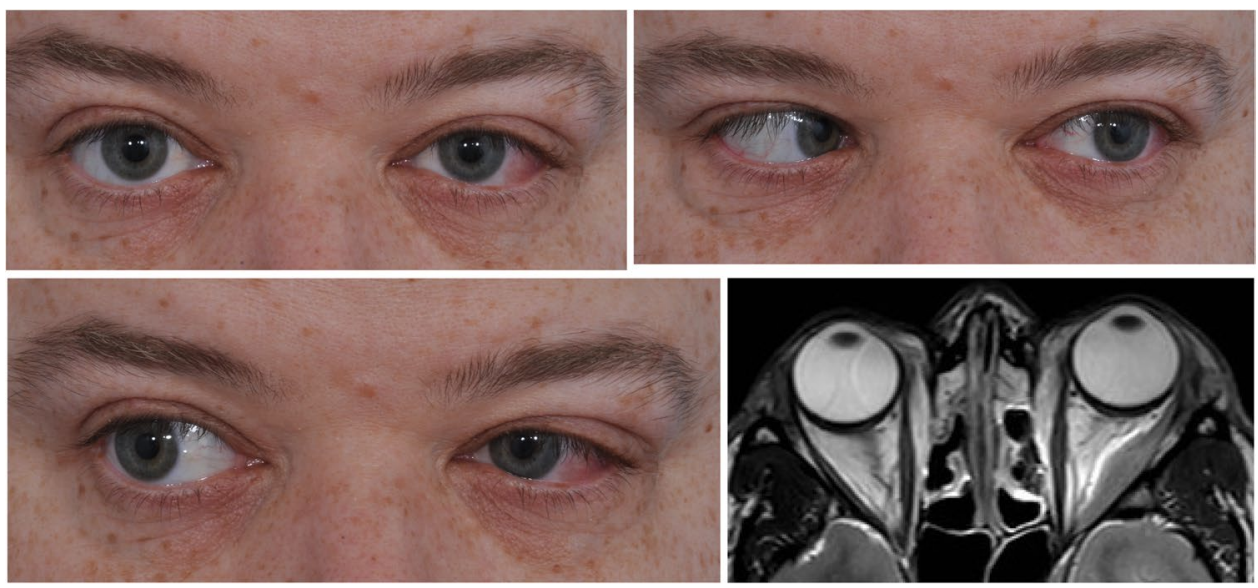
sign of IOM and may occur in infiltrative disease of the EOM or orbital fat, typically in metastasis from scirrous breast carcinoma.

\section{Imaging Findings}

The typical findings on imaging (computerized tomography (CT) or magnetic resonance imaging (MRI)) are homogeneous, fusiform enlargement of the muscle belly with enhancement, and often, but not always, involvement of the anterior muscle tendon (Fig. 1). Patrinely et al. reviewed the CT findings of patients with non-thyroid EOM enlargement, including a group of 12 patients with IOM [19]. Amongst these patients, 7 showed fusiform enlargement, and 4 had a cylindrical enlargement of the affected muscle. Sharp delineation of the affected muscle is usually observed in acute cases, but more chronic cases, and those with multiple muscle involvement and associated systemic disease tended to have less sharply defined borders, with spillover of the inflammatory process into the surrounding orbital fat. Most affected muscles were between 6 and $10 \mathrm{~mm}$ in diameter. Additional anterior tendon enlargement is observed in 41 to $57 \%$ of cases, but is not typical of IOM as it may also occur in giant cell myositis, lymphoproliferative disease, and, less frequently, in metastasis and severe thyroid eye disease [7, $8,12,16,20]$.

The presence of focal, nodular, or heterogeneous enlargement of an EOM is not typical of IOM and raises concerns about malignant pathology [20]. Additionally, the presence of disease of other orbital structures distant from the EOM should raise the possibility of other diagnoses [20]. Examples include (i) lacrimal gland enlargement in sarcoidosis, immunoglobulin G4 (IgG4)-related disease, and lymphoma; (ii) infra- or supra-orbital nerve enlargement in IgG4-related and reactive lymphoid hyperplasia; and (iii) orbital fat or optic nerve sheath involvement in lymphoma and breast carcinoma metastasis. Involvement of adjacent anatomical structures in continuity with the EOM, such as the lacrimal gland, orbital fat, eyelids, globe, conjunctiva, orbital bone, sinuses, or intracranial structures, represents locally invasive disease such as malignancy or infection, and may also occur in granulomatosis with polyangiitis. In such cases, tissue biopsy of the other structure, or of the enlarged muscle may secure the diagnosis. Similarly, scleritis may be associated with myositis of an adjacent muscle [21].

New MRI protocols include diffusion-weighted (DWI) and dynamic contrast-enhanced (DCE) MRI. They may help distinguishing malignant from benign orbital lesions, in particular high-grade lymphoma from inflammation and reactive lymphoid hyperplasia, although features of diseases may overlap [22, 23]. The use of Zirconium-89-labeled $\left({ }^{89} \mathrm{Zr}\right.$ )-rituximab positron emission tomography (PET-CT) has been studied in a small case series of therapy refractive IOM [24•]. The muscles exhibited low to moderate uptake of ${ }^{89} \mathrm{Zr}$-rituximab. Larger case studies, however, are required to assess the value of this novel method for B-cell imaging in demonstrating the cause of orbital myositis and predicting the therapeutic outcome with rituximab.

\section{Muscle Biopsy}

Tissue biopsy may be of importance in demonstrating the possible cause of orbital myositis. Indications are atypical or inconclusive clinico-radiological findings for a diagnosis of IOM, or where the clinical behavior changes or fails to respond to treatment $[25 \bullet, 26]$. Atypical cases include the absence of pain, slow onset of symptoms, all EOMs affected, focal, nodular or inhomogeneous muscle enlargement, extremely enlarged single or multiple EOMs, other (either distant from or adjacent to the EOM) orbital or periorbital structures involved, history of primary or secondary malignancy, and unresponsiveness to corticosteroids [20]. The main aim to proceed with a muscle biopsy is excluding other disease.

The muscle biopsy is obtained through a minimally invasive surgical approach. The anterior part of the muscle can be accessed using a strabismus surgery approach with the incision placed in the conjunctiva of the eye. Where the biopsy needs to be directed to the posterior half of the muscle, access is achieved via a transconjunctival or transcutaneous orbitotomy procedure [20]. These are minimally invasive procedures, and they often can be performed with the patient under local anesthesia. However, in the dissection or sampling process of biopsy of the posterior part of a muscle, damage may occur to the muscle or its motor innervation.

The biopsy specimen should be representatively procured from the involved site and sufficient in size for preparing possible ancillary testing with immunohistochemistry, immunophenotyping, and gene rearrangement in addition to routine hematoxylin-eosin staining for histomorphologi-

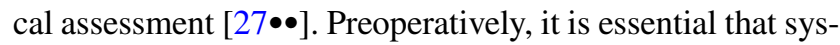
temic or intralesional corticosteroids are avoided for at least 2 weeks prior to obtaining the biopsy as they may affect the immunological picture on histopathology and its interpretation. There is little published data on the histopathological findings in IOM. The largest study to date examined the features in 9 definite cases of IOM [15•]. These cases were biopsied because of atypical features and therefore may not be representative of typical forms of IOM where the presentation and response to treatment with systemic corticosteroids preclude the need for a biopsy. Nonetheless, the study provides useful information. Two-thirds of cases were female, 7/9 had a single affected muscle, and the medial rectus was the most commonly biopsied muscle. Muscle fibers 
were splayed by inflammatory infiltrates which consisted of lymphocytes (predominantly B cells) (9/9), plasma cells (6/9), and histiocytes (6/9). Eosinophils (4/9), polymorphs (1/9), and giant cells (1/9) were much less frequent. Muscle fiber degeneration and regeneration were seen in 5/9 cases.

The presence of granulomatous inflammation with giant cells on biopsy should raise the possibility of another diagnosis than IOM, such as sarcoidosis or giant cell myositis [28]. The latter is associated with giant cell myocarditis, a potentially fatal condition. Giant cell orbital myositis may precede the development of cardiac symptoms by sometimes many months [29]. It is often associated with a constellation of auto-immune phenomena, including vitiligo [28].

\section{Differential Diagnosis}

Many other diseases with inflammation or infiltration of EOMs can occur and these may mimic IOM. Additionally, orbital myositis may develop in the setting of specific inflammatory or auto-immune conditions. Table 1 provides a simplified classification of all forms of orbital myositis $[3 \bullet \bullet]$. The list of causes is not limitative, considering a recently reported case with presumed orbital myositis following SARS-CoV19 infection [30].

By far, the commonest auto-immune condition associated with inflammation of the EOMs is GO. A review of GO is beyond the scope of this paper, but in all patients presenting with EOM enlargement, clinical features of GO should be sought: features of thyroid gland dysfunction, and other ocular features of GO, in particular upper lid retraction and lid lag on down gaze. Additionally, thyroid function should be tested and auto-antibodies, particularly TSH (thyroid stimulating hormone) receptor antibodies, should be measured.

Table 1 List of clinical forms of orbital myositis

\begin{tabular}{l}
\hline Idiopathic \\
- Acute, single muscle \\
- Acute, multiple muscles \\
- Subacute \\
- Recurrent \\
- Chronic \\
Related to systemic or local conditions \\
- Infective \\
- Bacterial \\
- Viral \\
- Parasitic \\
- Associated with auto-immune diseases (includes Graves' orbit- \\
$\quad$ opathy) \\
- Associated with systemic or local specific inflammatory diseases \\
Drug reactions \\
Paraneoplastic \\
\hline
\end{tabular}

GO may affect any or many EOMs, but the relative frequency of involvement of EOMs varies, and the relative order from most frequent to least is inferior rectus $>$ medial rectus $>$ superior rectus/levator $>$ lateral rectus $>$ obliques, which is different to the order of IOM.

Orbital myositis has been reported in a large range of non-thyroid auto-immune and specific inflammatory conditions (Table 2). This range of conditions, as well as many of the types of conditions listed in Table 1 are also associated with uveitis and scleritis, and a similar investigative approach may be appropriate in patients with IOM as is used in patients with uveitis and scleritis [3••]. Patients with orbital myositis occurring in association with these conditions tend to much more frequently have multiple EOMs affected, and bilateral disease is also much more common. The presentation is often more subacute or chronic, and pain is less commonly a feature. Importantly, orbital myositis may present prior to the development of some of these conditions such as inflammatory bowel disease and giant cell myocarditis [29, 31]. Some of the associated conditions will manifest other orbital features of the disease, such as

Table 2 Auto-immune and inflammatory conditions associated with orbital myositis (in order of reported frequency)

\author{
Graves' disease \\ IgG4-related disease \\ Inflammatory bowel disease \\ Sarcoidosis \\ SLE and DLE \\ Giant cell myocarditis \\ Post-streptococcal pharyngitis \\ Rheumatoid arthritis \\ EGPA \\ Behçet's disease \\ GPA \\ Psoriatic arthritis/psoriasis \\ Sjögren's syndrome \\ Dermatomyositis \\ HLA-B27 spondyloarthritis \\ Scleroderma \\ Celiac disease \\ Primary biliary cirrhosis \\ Kawasaki's disease \\ Relapsing polychondritis \\ NOD-2 autoimmune disease
}

SLE systemic lupus erythematosus, $D L E$ discoid lupus erythematosus, EGPA eosinophilic granulomatosis with polyangiitis (Churg Strauss syndrome), GPA granulomatosis with polyangiitis (Wegener's granulomatosis), NOD-2 nucleotide-binding oligomerization domain 2 (NOD2) gene mutations 
lacrimal gland involvement in sarcoidosis and IgG4-related disease. Long-term follow-up may identify associated systemic pathologies.

Many non-inflammatory conditions can cause EOM enlargement, and these are classified as (i) neoplastic (e.g., lymphoma; metastases); (ii) deposition (e.g., amyloidosis); and (iii) vascular (e.g., orbital venous congestion (e.g., from carotid-cavernous sinus fistula)); muscle hematoma) [20].

\section{Treatment}

Management is tailored to severity and progression of disease. Mild cases, i.e., monomuscular, mild pain, and normal or discretely impaired ocular motility, can be treated with high-dose of nonsteroidal anti-inflammatory drugs, with a slow taper over several weeks. Corticosteroids are the preferred treatment in moderate to severe cases with high morbidity and strabismus. Patients with IOM typically show dramatic improvement of their signs and symptoms within $48 \mathrm{~h}$ after systemic administration of prednisolone at an initial dose of $1 \mathrm{mg}$ per kg body weight, referred to as the corticosteroid trial as it may also confer diagnostic sensitivity for IOM [26, 32]. The administration is intravenously or orally. Following initial high dose, systemic steroid administration should be tapered off gradually over several months. There is anectodal evidence of initial benefit with perilesional triamcinolone injection as an alternative to systemic administration in IOM [33].

However, relapse on tapering or ceasing of the antiinflammatory drugs with recurrences months or years after the episode is common. Reported recurrence rates vary between 23\% (in a series of 26 cases) and 35\% (in a series of 26 patients) and $56 \%$ (in a series of 16 patients, one or more recurrence with a disease-free interval of just 4.1 months (range 0.5-17 months)) [7-9]. Features associated with recurrence were male gender, multiple involved EOMs, and a lack of response to corticosteroids [7,9].

In corticosteroid-dependent and recalcitrant IOM, nonspecific immunosuppressive and specific biologicals can be used as second- and third-line therapy to control the inflammatory process, with varying degrees of success. The antimetabolite methotrexate is moderately successful and welltolerated as corticosteroid-sparing agent in idiopathic orbital inflammation, and should be given for a prolonged time [34, $35 \bullet \bullet$. Infliximab, a biologic anti-tumor necrosis factor-alpha agent, has anti-inflammatory potential in IOM cases which are severe, recurrent, or recalcitrant to corticosteroids and methotrexate [36]. A cortico-dependent case of IOM was successfully treated with the monoclonal anti-interleukin-6 receptor antibody tocilizumab [37]. In view of their systemic toxicity profile, these drugs are reserved for chronic or recurrent IOM with severe symptomatology.

IOM displays a low radiosensitivity. Low-dose orbital irradiation delivered in the acute phase of recurrent IOM cannot prevent but only delay a next recurrence $[8,38]$.

Chronic orbital myositis is commoner in patients with a poor response to corticosteroids, and this should raise concerns about the diagnosis. A biopsy should be obtained to exclude other pathologies. In patients with a chronic course, many will have an underlying systemic disease, and may require corticosteroid-sparing or biological agents [36]. In all such atypical cases, a long-term follow-up may identify associated systemic pathologies.

The management of diplopia depends on the stage of the disease. Monocular occlusion is the simplest measure to relieve diplopia. A Fresnel prism pressed on to the back surface of the prescription glasses is a temporary measure where double vision is expected to recover over time, or as a trial before a permanent prism can be built into new glasses. Alternatively, Botulinum toxin (BTXA) can be injected in a contracted muscle to improve diplopia in selected cases of IOM [39]. When permanent strabismus has settled in the inactive phase, strabismus surgery is carried out to align the eyes and hence restore the field of binocular single vision.

\section{Conclusion}

IOM is a rare disease characterized by acutely inflamed and enlarged EOMs from unknown origin. With advanced identification and classification of orbital disorders, the concept of IOM is reduced to cases without associated local or systemic disease. The diagnosis is supported by the clinical triad of acute onset of orbital pain exacerbated on eye movement, double vision, and peri-ocular redness or swelling, with radiological evidence of homogeneous enlargement and enhancement of one or more EOMs, with or without the involvement of the anterior tendon. IOM typically responds well to high-dose systemic corticosteroids which are given for a prolonged time with slow taper to prevent recurrent or chronic disease.

IOM is not a sound diagnosis as it has features overlapping those of other diseases. When the clinico-radiological pattern deviates from the classical IOM pattern, systemic and oncologic screening and obtaining muscle biopsy are warranted. IOM remains at length a diagnosis of exclusion for the clinician, radiologist, and pathologist.

\section{Compliance with Ethical Standards}

Conflict of Interest The authors declare no competing interests. 
Human and Animal Rights All reported studies/experiments with human or animal subjects performed by the authors have been previously published and complied with all applicable ethical standards (including the Helsinki declaration and its amendments, institutional/ national research committee standards, and international/national/institutional guidelines).

Informed Consent Additional informed consent was obtained from the individual participant for whom identifying information is included in this article.

\section{References}

Papers of particular interest, published recently, have been highlighted as:

- Of importance

$\bullet$ Of major importance

1. Verma M, Fitzpatrick K, McLoon LK. Extraocular muscle repair and regeneration. Curr Ophthalmol Rep. 2017;5:207-15. https:// doi.org/10.1007/s40135-017-0141-4.

2. Yu Wai Man CY, Chinnery PF, Griffiths PG. Extraocular muscles have fundamentally distinct properties that make them selectively vulnerable to certain disorders. Neuromuscular disorders 2005;15:17-23. https://doi.org/10.1016/j.nmd.2004.10. 002 .

3. McNab AA. Orbital myositis: a comprehensive review and reclassification. Ophthalmic Plast Reconstr Surg. 2020;36:109 17. https://doi.org/10.1097/IOP.0000000000001429. Comprehensive review of the various causes of orbital myositis emphasizing the need of a systematic approach to nomenclature, diagnosis and treatment.

4. Gleason JE. Idiopathic myositis involving the extraocular muscles. Am J Ophthalmol. 1903;12:471-8.

5. Birsh-Hirschfeld A. Zur diagnostik und pathologie der orbitaltumoren. Ber Dtsch Ophthalmol Ges. 1905;32:127-35.

6. Mombaerts I, Goldschmeding R, Schlingemann RO, Koornneef L. What is orbital pseudotumor? Surv Ophthalmol. 1996;4:6678. https://doi.org/10.1016/s0039-6257(97)81996-0.

7. Mannor GE, Rose GE, Moseley IF, Wright JE. Outcome of orbital myositis clinical features associated with recurrence. Ophthalmology. 1997;104:409-14. https://doi.org/10.1016/ s0161-6420(97)30300-5.

8. Mombaerts I, Koornneef L. Current status in the treatment of orbital myositis. Ophthalmology. 1997;104:402-8. https://doi. org/10.1016/s0161-6420(97)30301-7.

9. Kang MS, Yang HK, Kim N, Hwang JM. Clinical features of ocular motility in idiopathic orbital myositis. J Clin Med. 2020;18(9):1165. https://doi.org/10.3390/jcm9041165.

10. Jakobiec FA, Syed ZA, Stagner AM, Harris GJ, Rootman J, Yoon $\mathrm{MK}$, et al. Orbital inflammation in pregnant women. Am J Ophthalmol. 2016;166:91-102. https://doi.org/10.1016/j.ajo.2016. 03.029 .

11. Scott IU, Siatkowski RM. Idiopathic orbital myositis. Curr Opinion. Rheumatol. 1997;9:504-12. https://doi.org/10.1097/00002 281-199711000-00005.

12. Yan JY, Wu P. Idiopathic orbital myositis. J Craniofac Surg. 2014;25:884-7. https://doi.org/10.1097/SCS.0000000000 000510 .

13. Avni-Zauberman N, Tripathy D, Rosen N, Ben Simon GJ. Relapsing migratory idiopathic orbital inflammation: six new cases and review of the literature. Br J Ophthalmol. 2012;96:276-80. https://doi.org/10.1136/bjo.2010.191866.

14. Siatkowski RM, Capo H, Byrnse SF, Gendron EK, Flynn JT, Muñoz M, et al. Clinical and echographic findings in idiopathic orbital myositis. Am J Ophthalmol. 1994;118:343-50. https://doi.org/10.1016/s0002-9394(14)72959-9.

15. Ben Artsi E, McKelvie PA, McNab AA. Histopathological findings in idiopathic orbital myositis. Ophthalmology. 2021;128:609-16. https://doi.org/10.1016/j.ophtha.2020.09. 012. Rare case study on the histopathological spectrum of idiopathic orbital myositis.

16. Mombaerts I, Tousseyn T, Van Limbergen E, Demaerel P. Clinically recognizing enlarged extraocular muscles from lymphoid origin. Ophthalmology. 2015;122:217-8. https:// doi.org/10.1016/j.ophtha.2014.08.018.

17. Moorman CM, Elston JS. Acute orbital myositis Eye. 1995;9:96-101. https://doi.org/10.1038/eye.1995.15.

18. Osher RH, Schatz NJ, Duane TD. Acquired orbital retraction syndrome. Arch Ophthalmol. 1980;98:1798-802. https://doi. org/10.1001/archopht.1980.01020040650012.

19. Patrinely JR, Osborn AG, Anderson RL, Whiting AS. Computed tomographic features of nonthyroid extraocular muscle enlargement. Ophthalmology. 1989;96:1038-47. https://doi. org/10.1016/s0161-6420(89)32785-0.

20. Mombaerts I, Rose GE, Verity DH. Diagnosis of enlarged extraocular muscles: when and how to biopsy. Curr Opin Ophthalmol. 2017;28:514-21. https://doi.org/10.1097/ICU.00000 00000000395 .

21. Boonman ZF, De Keizer RJ, Graniewski-Wijnands HS, Watson PG. Orbital myositis in scleritis. Br J Ophthalmol. 2003;87:38-42. https://doi.org/10.1136/bjo.87.1.38.

22. Fatima Z, Ichikawa T, Ishigame K, Motosugi U, Waqar AB, Hori M, et al. Orbital masses: the usefulness of diffusionweighted imaging in lesion categorization. Clin Neuroradiol. 2014;24:129-34. https://doi.org/10.1007/s00062-013-0234-X.

23. Hu H, Xu XQ, Liu H, Hong XN, Shi HB, Wu FY. Orbital benign and malignant lymphoproliferative disorders: differentiation using semi-quantitative and quantitative analysis of dynamic contrast-enhanced magnetic resonance imaging. Eur J Radiol. 2017;88:88-94. https://doi.org/10.1016/j.ejrad.2016. 12.03 .

24. Laban KG, Kalmann R, Leguit RJ. de Keizer B 2019 Zirconium89-labelled rituximab PET-CT in orbital inflammatory disease. EJNMMI Res. 2019;9:1-7. https://doi.org/10.1186/s13550019-0530-9. Pilot study on a novel B-cell imaging method in patients with non-specific and specific orbital inflammatory disease.

25. Eade EL, Hardy TG, McKelvie PA, McNab AA. Review of extraocular muscle biopsies and utility of biopsy in extraocular muscle enlargement. Br J Ophthalmol. 2018;102:1586-90. https://doi.org/10.1097/IOP.0000000000001426. Large case study on biopsy-proven benign and malignant extraocular muscle disease, highlighting the need of muscle biopsy.

26. Mombaerts I, Bilyk JR, Rose GE, McNab AA, Fay A, Dolman $\mathrm{PJ}$, et al. Consensus on diagnostic criteria of idiopathic orbital inflammation using a modified Delphi approach. JAMA Ophthalmol. 2017;135:769-76. https://doi.org/10.1001/jamaophtha lmol.2017.1581.

27. Mombaerts I, Ramberg I, Coupland SE, Heegaard S. Diagnosis of orbital mass lesions: clinical, radiological, and pathological recommendations. Surv Ophthalmol. 2019;64:741-56. https:// doi.org/10.1016/j.survophthal.2019.06.006. Review of the diagnostic algorithm of orbital mass lesions with emphasis on the tissue biopsy.

28. Selva D, Dolman PJ, Rootman J. Orbital granulomatous giant cell myositis: a case report and review. Clin Exp Ophthalmol. 
2000;28:65-8. https://doi.org/10.1046/j.1442-9071.2000. 00273.x.

29. Kattah JC, Zimmerman LE, Kolsky MP, Chrousos G, Chavis $\mathrm{R}$, Jaffe $\mathrm{G}$, et al. Bilateral orbital involvement in fatal giant cell polymyositis. Ophthalmology. 1990;97:520-5. https://doi.org/ 10.1016/s0161-6420(90)32554-x.

30. Armstrong BK, Murchison AP, Bilyk JR. Suspected orbital myositis associated with COVID-19. Orbit. 2021;40:532-5. https://doi.org/10.1080/01676830.2021.1962366.

31. Vargason CW, Mawn LA. Orbital myositis as both a presenting and associated extraintestinal sign of Crohn's disease. Ophthalmic Plast Reconstr Surg. 2017;33(3S Suppl 1):S158-60. https:// doi.org/10.1097/IOP.0000000000000475.

32. Glass LRD, Freitag SK. Orbital inflammation: corticosteroids first. Surv Ophthalmol. 2016;6:670-3. https://doi.org/10.1016/j. survophthal.2016.01.005.

33. Leibovitch I, Prabhakaran VC, Davis G, Selva D. Intraorbital injection of triamcinolone acetonide in patients with idiopathic orbital inflammation. Arch Ophthalmol. 2007;125:1647-51. https://doi.org/10.1001/archopht.125.12.1647.

34. Smith JR, Rosenbaum JT. A role for methotrexate in the management of non-infectious orbital inflammatory disease. Br J Ophthalmol. 2001;85:1220-4. https://doi.org/10.1136/bjo.85. 10.1220 .

35. Lee MJ, Planck SR, Choi D, Harrington CA, Wilson DJ, Dailey RA, et al. Non-specific orbital inflammation: current understanding and unmet needs. Prog Retin Eye Res. 2021;81:100885. https://doi.org/10.1016/j.preteyeres.2020. 100885. Comprehensive overview of the current state of clinical knowledge regarding non-specific orbital inflammation.

36. Garrity JA, Coleman AW, Matteson EL, Eggenberger ER, Waitzman DM. Treatment of recalcitrant idiopathic orbital inflammation (chronic orbital myositis) with infliximab. Am J Ophthalmol. 2004;138:925-30. https://doi.org/10.1016/j.ajo.2004.06. 077.

37. Meca Casasnovas N, Sangrador Pelluz C, Sotoca J. Tocilizumab as an alternative for corticosteroid-refractory orbital myositis. J Neuroimmunol. 2021;356: 577573. https://doi.org/10.1016/j. jneuroim.2021.577573.

38. Isobe K, Uno T, Kawakami H, Ueno N, Kawata T, Abe H, et al. Radiation therapy for idiopathic orbital myositis: two case reports and literature review. Radiat Med. 2004;22:429-31.

39. Bessant DA, Lee JP. Management of strabismus due to orbital myositis. Eye (Lond). 1995;9(Pt 5):558-63. https://doi.org/10. 1038/eye.1995.139.

Publisher's Note Springer Nature remains neutral with regard to jurisdictional claims in published maps and institutional affiliations. 